\title{
Percepção da imagem corporal e fatores associados em idosos residentes em município do nordeste brasileiro: um estudo populacional
}

\author{
Body image perception and associated factors among elderly residents \\ in a city in northeast Brazil: a population-based study
}

Tarciana Nobre de Menezes ${ }^{1}$

Kyonayra Quezia Duarte Brito ${ }^{1}$

Elaine Cristina Tôrres Oliveira ${ }^{1}$

Dixis Figueroa Pedraza ${ }^{1}$

${ }^{1}$ Conselho de Ensino, Pesquisa e Extensão, Universidade Estadual da Paraíba. R. Baraúnas 351, Bodocongó. 58.429-500 Campina Grande PB Brasil tnmenezes@hotmail.com.br

\begin{abstract}
The article aims to verify body image perception and associated factors among elderly individuals in Campina Grande, State of Paraiba. It involves a cross-sectional, population and home-based study, with individuals 60 years or older of both sexes. Body image perception was considered the dependent variable for purposes of analysis and study of possible associations. The independent variables were: age group, nutritional status, number of diseases, health perception and regular practice of physical activities. To identify associated factors, univariate and multivariate regression analyses were carried out using SPSS 17.0 software. Overall, 806 elderly individuals were interviewed. Men showed greater satisfaction with body image when compared to women and older participants were more satisfied than younger participants. Among the factors related to dissatisfaction in women, underweight and overweight/ obesity were associated with an increased risk of dissatisfaction. Among men, older age constituted a protective factor for dissatisfaction, while underweight and overweight/obesity were risk factors for dissatisfaction. The results of this study suggest a possible influence of nutritional status on body image perception among the elderly, negatively affecting their perception of body image.
\end{abstract}

Key words Elderly, Health, Body image perception
Resumo O objetivo do artigo é verificar a percepção da imagem corporal e fatores associados em idosos residentes em Campina Grande (PB). Trata-se de um estudo transversal, de base populacional e domiciliar, com indivíduos de 60 anos ou mais, de ambos os sexos. A percepção da imagem corporal foi considerada variável dependente para fins de análise e de estudo das possíveis associações. As variáveis independentes avaliadas foram: grupo etário, estado nutricional, número de doenças, percepção de saúde e prática de atividade física regular. Para identificar os fatores associados foram realizadas análises de regressão univariada e multivariada, por meio do SPSS 17.0. Foram avaliados 806 idosos. Os homens apresentaram maior satisfação com a imagem corporal quando comparados às mulheres, e idosos mais velhos apresentaram-se mais satisfeitos que idosos mais jovens. Entre os fatores relacionados à insatisfação nas mulheres, o baixo peso e sobrepeso/obesidade estavam relacionados a um risco aumentado de insatisfação. Entre os homens, idades avançadas constituíram fator de proteção para insatisfação, enquanto baixo peso e sobrepeso/obesidade foram fatores de risco para insatisfação. Os resultados sugerem possível influência do estado nutricional na percepção da imagem corporal, interferindo negativamente na percepção da imagem.

Palavras-chave Idoso, Saúde, Percepção da imagem corporal 


\section{Introdução}

Durante o envelhecimento, o indivíduo é acometido, de forma dinâmica e progressiva, por alterações biopsicossociais que predispõem a uma diminuição da capacidade de adaptação ao meio ambiente, maior vulnerabilidade e maior incidência de agravos à saúde ${ }^{1-3}$. Entre as alterações ocorridas nesse período, às relacionadas à flacidez da pele e músculos, perda do brilho e cor dos cabelos e aumento do peso, constituem-se motivos para a insatisfação e preocupação com a imagem corporal entre os idosos ${ }^{4}$. Todavia, a imagem corporal envolve um conjunto complexo de fatores psicológicos, sociais, culturais e biológicos associados a mudanças ocorridas durante o envelhecimento ${ }^{5}$. Devido a isso, torna-se importante conhecer como esses indivíduos percebem essas mudanças, de que forma interferem na percepção da imagem corporal e como influenciam a vida dos mesmos ${ }^{6}$.

A percepção da imagem corporal é uma representação mental que o indivíduo tem do seu próprio corpo e que pode influenciar o seu estado geral de saúde ${ }^{7}$. Considerando que cada indivíduo envelhece de modo particular, alguns aspectos específicos podem interferir na concepção que os idosos têm da sua imagem corporal, como é o caso do sexo, idade ${ }^{8}$, estado nutricional ${ }^{9,10}$, nível de atividade física ${ }^{9}$, número de doenças ${ }^{11}$ e percepção da saúde ${ }^{12}$.

Estudos específicos com idosos objetivando avaliar a percepção da imagem corporal têm sido realizados internacionalmente ${ }^{13-15}$, assim como no Brasil, com idosos institucionalizados ${ }^{7,11}$, idosos participantes de grupos de convivência ${ }^{10,16}$ e idosos praticantes de atividade física ${ }^{17,18}$. Esses estudos têm verificado elevada prevalência de indivíduos insatisfeitos com sua imagem corporal ${ }^{10,19,20}$, relacionando essa insatisfação ao excesso de peso ${ }^{21}$, às doenças vivenciadas 9 , à avaliação negativa da saúde ${ }^{12}$, à prática de atividades físicas regulares ${ }^{22,23}$ e ao estado nutricional não adequado ${ }^{9,10}$.

Nesse contexto, considerando a importância de se observar a percepção que os idosos fazem do seu corpo, assim como os fatores que a influenciariam, o presente estudo teve como objetivo verificar a percepção da imagem corporal dos idosos residentes no município de Campina Grande/PB e sua associação com o estado nutricional, número de doenças, percepção do estado de saúde e prática de atividade física regular.

\section{Material e Métodos}

Trata-se de um estudo de base populacional, domiciliar, do tipo transversal, com coleta de dados primários. A população é constituída por indivíduos com 60 anos ou mais, de ambos os sexos, residentes habituais da cidade de Campina Grande (PB). Foram excluídos do estudo idosos que apresentassem debilidade clínica grave, sem possibilidades terapêuticas; e os idosos que estivessem ausentes de Campina Grande por mais tempo que a pesquisa de campo no setor censitário de residência do idoso.

Para o cálculo da amostra foi utilizado uma estimativa de prevalência dos desfechos de, no mínino, 50\%, com limite de confiança de $95 \%$, admitindo-se um erro de $5 \%$ e correção amostral de 2,1, resultando em uma amostra de 806 idosos.

A amostra deste estudo foi constituída por idosos residentes em 24 setores censitários, dos seis Distritos Sanitários, da cidade de Campina Grande. Para a seleção da amostra foi utilizada a metodologia adotada pelo Instituto Brasileiro de Geografia e Estatística (IBGE), que divide as cidades em setores censitários, os quais foram obtidos de forma aleatória.

A obtenção da amostra ocorreu da seguinte forma: todos os quarteirões de cada setor censitário foram numerados, para posterior sorteio. Após escolha aleatória do quarteirão, a identificação do primeiro domicílio ocorreu considerando como ponto de partida a esquina inicial do setor, em sentido horário. A cada domicílio selecionado, foi saltado um número de nove domicílios, de acordo com cada setor censitário, e assim sucessivamente, a fim de obter-se melhor distribuição. Foi considerado domicílio o local utilizado como habitação de uma ou mais pessoas. Os locais constituídos por estabelecimentos coletivos como hospitais, clínicas, escolas, clubes, quartéis e similares, mesmo que destinados aos idosos, foram excluídos da seleção aleatória, por conseguinte, do estudo.

Se no domicílio selecionado não residisse idoso ou se o mesmo não aceitasse ou não pudesse participar do estudo, outro idoso foi procurado no domicílio mais próximo. No caso de residir mais de um idoso no mesmo local, todos foram convidados a participar do estudo. Quando residia idoso no domicílio, porém o mesmo estava ausente, eram realizadas até mais duas visitas de retorno. 
O trabalho de campo foi realizado por três duplas de entrevistadores, os quais eram alunos (voluntários e bolsistas) de cursos de graduação da área da saúde e foram treinados pela coordenadora do estudo.

A variável percepção da imagem corporal foi considerada como variável dependente para fins de análise e de estudo das possíveis associações. A imagem corporal foi verificada por meio da escala de nove silhuetas de Stunkard et al. ${ }^{24}$, onde o idoso foi orientado a observar a escala e identificar a silhueta que melhor representasse sua aparência física atualmente. Após a resposta, o idoso era questionado se gostaria de ter a silhueta apontada ou gostaria de ter outra. Foi considerado idoso satisfeito com a imagem corporal aquele que gostaria de ter a silhueta apontada. Dessa forma, os idosos foram classificados como satisfeitos ou insatisfeitos com a imagem corporal.

As variáveis independentes avaliadas foram:

- Grupo etário: definidos como 60 a 69 anos, 70 a 79 anos e 80 anos ou mais.

- Estado nutricional: a avaliação do estado nutricional foi realizada por meio do Índice de Massa Corporal (IMC) $\left(\mathrm{Kg} / \mathrm{m}^{2}\right)$, classificado de acordo com os valores propostos pela Organização Pan-Americana de Saúde (OPAS): baixo peso $\left(\right.$ IMC $<23 \mathrm{Kg} / \mathrm{m}^{2}$ ), eutrofia (IMC $\geq 23 \mathrm{Kg} / \mathrm{m}^{2} \mathrm{e}$ IMC $<28 \mathrm{Kg} / \mathrm{m}^{2}$ ), sobrepeso (IMC $\geq 28 \mathrm{Kg} / \mathrm{m}^{2}$ e IMC $<30 \mathrm{Kg} / \mathrm{m}^{2}$ ) e obesidade (IMC $\geq 30 \mathrm{Kg} /$ $\left.\mathrm{m}^{2}\right)^{25}$. Para fins estatísticos as categorias de sobrepeso e obesidade foram agrupadas, sendo consideradas em uma única categoria: sobrepeso/obesidade. Para o cálculo do IMC, que consiste na razão entre o peso e o quadrado da estatura $(\mathrm{kg} /$ $\mathrm{m}^{2}$ ), foram aferidos o peso e a estatura, de acordo com as técnicas propostas por Gordon et al. ${ }^{26}$.

- Número de doenças: obtida por meio da pergunta Um médico ou profissional de saúde the disse que tem alguma dessas doenças?, tendo o idoso oito opções de respostas (pressão alta, artrite/artrose/reumatismo, problema cardíaco, diabetes, osteoporose, doença crônica pulmonar (DCP), embolia/derrame, tumor maligno). O número de doenças foi categorizado em: nenhuma, 1, 2 ou 3,4 ou mais.

- Percepção da saúde: obtida por meio do questionamento Como o senhor(a) considera a sua saúde?, tendo como opção de resposta excelente, muito boa, boa, regular e má. Para fins estatísticos as categorias de percepção da saúde foram categorizadas como: boa (excelente, muito boa, boa) e ruim (regular e má).

- Prática de atividade física regular: foi considerado idoso ativo aquele que praticava algum tipo de atividade física ao menos três vezes por semana, por no mínimo trinta minutos. Sendo assim, os idosos foram classificados como realizando ou não atividade física regular.

\section{Análise Estatística}

Foram verificadas as proporções de idosos satisfeitos e insatisfeitos com a imagem corporal de acordo com o sexo e grupo etário. A associação entre percepção da imagem corporal, sexo e grupo etário foi verificada por meio do Teste de Qui-quadrado de Pearson $\left(\chi^{2}\right)$. Para identificação dos fatores associados com a percepção da imagem corporal, foram realizadas análises de regressão univariada, para cálculo dos odds ratios (OR) brutos, com intervalo de confiança (IC) de 95\%. Para elaboração do modelo final, foi utilizada análise de regressão logística multivariada, com o método de inclusão passo a passo (forward stepwise), para o cálculo do OR ajustado, no qual foram selecionadas as variáveis que obtiveram um $\mathrm{p} \leq$ 0,20 , a partir do modelo de regressão univariada. As informações estatísticas foram obtidas com o auxílio do aplicativo estatístico Statistical Package for Social Sciences (SPSS) versão 17.0.

\section{Aspectos éticos}

Todos os idosos assinaram o Termo de Consentimento Livre e Esclarecido depois de receberem explicações verbais e escritas a respeito do estudo. O estudo maior, do qual este faz parte, foi aprovado pelo Comitê de Ética em Pesquisa da Universidade Estadual da Paraíba (UEPB), não havendo conflito de interesses.

\section{Resultados}

Foram entrevistados 806 idosos $(69,1 \%)$, com idade que variou de 60 a 103 anos, com média de 72,4 anos (DP = 8,76). Dos 806 idosos estudados, não foi possível calcular o IMC de 23 idosos, tendo em vista a impossibilidade ou dificuldades de aferição do peso e/ou estatura dos mesmos. $\mathrm{Na}$ Tabela 1 é possível observar a distribuição dos idosos de acordo com a percepção da imagem corporal, sexo e grupo etário. Foi verificado que $51,3 \%$ das mulheres e $68,0 \%$ dos homens estavam satisfeitos com sua imagem corporal. Foi verificada associação estatisticamente significativa entre percepção da imagem corporal e sexo ( $\mathrm{p}<$ 0,001 ). Quanto ao grupo etário é possível observar que a maior proporção de idosos satisfeitos 
era do grupo com 80 anos ou mais. Foi verificada associação estatisticamente significativa entre percepção da imagem corporal e grupo etário ( $\mathrm{p}$ $<0,05)$.

Apesar da elevada prevalência de idosos satisfeitos com a imagem corporal, é preciso observar os idosos insatisfeitos, visto que a insatisfação com a imagem corporal pode estar associada ao estado geral de saúde.

Tabela 1. Distribuição dos idosos de acordo com a percepção da imagem corporal (PIC), sexo e grupo etário. Campina Grande/PB, Brasil.

\begin{tabular}{lccc}
\hline \multirow{2}{*}{ Variáveis } & \multicolumn{3}{c}{$\begin{array}{c}\text { Percepção da imagem corporal } \\
\text { (\%) }\end{array}$} \\
\cline { 2 - 4 } & $\mathbf{N}$ & Satisfeito & Insatisfeito \\
\hline Sexo & & & \\
Masculino & 241 & 68,0 & 32,0 \\
Feminino & 542 & 51,3 & 48,7 \\
Grupo Etário* & & & \\
60 a 69 anos & 349 & 53,6 & 46,4 \\
70 a 79 anos & 270 & 53,3 & 46,7 \\
80 anos ou mais & 164 & 67,7 & 32,3 \\
\hline
\end{tabular}

Na Tabela 2 são apresentados os valores das análises univariadas entre a percepção da imagem corporal e os fatores associados estudados entre as mulheres, assim como os valores dos odds ratio brutos. Observou-se que entre as idosas insatisfeitas com a imagem corporal as com 60 a 69 anos e 70 a 79 anos apresentaram prevalências semelhantes $(50,4 \%$ e $50,8 \%$, respectivamente). Além disso, a maior prevalência de insatisfação esteve entre aquelas com sobrepeso/ obesidade $(65,8 \%)$, que referiram quatro ou mais tipos de doenças $(57,5 \%)$, com percepção de sua saúde ruim $(50,4 \%)$ e com prática de atividade física regular (50,5\%). Foi observada associação estatisticamente significativa apenas entre percepção da imagem corporal e estado nutricional.

Entre as mulheres, tanto aquelas com baixo peso como as com sobrepeso/obesidade apresentavam risco aumentado de estarem insatisfeitas com sua imagem corporal quando comparadas às eutróficas, tendo em vista que as com baixo peso apresentavam 3,51 vezes mais chances e as com sobrepeso/obesidade 3,11 vezes mais chances de insatisfação com a imagem.

Na Tabela 3 são apresentados os valores das análises univariadas entre a percepção da imagem corporal e os fatores associados estudados, assim como os valores dos odds ratio brutos entre os

Tabela 2. Regressão univariada em relação ao desfecho percepção da imagem corporal insatisfeita e fatores associados (grupo etário, estado nutricional, $\mathrm{n}^{\circ}$ de doenças, percepção de saúde, prática de atividade física regular (AFR)) entre as mulheres. Campina Grande (PB), Brasil.

\begin{tabular}{|c|c|c|c|c|}
\hline Variáveis & $\mathbf{n}$ & $\begin{array}{l}\text { Percepção da imagem } \\
\text { corporal insatisfeita (\%) }\end{array}$ & $\mathrm{p}(\mathrm{IC}$ 95\%) & OR \\
\hline \multicolumn{5}{|l|}{ Grupo etário } \\
\hline 60 a 69 anos & 127 & 50,4 & - & 1,0 \\
\hline 70 a 79 anos & 93 & 50,8 & $0,108(0,435-1,086)$ & 0,687 \\
\hline 80 anos ou mais & 44 & 41,1 & $0,111(0,417-1,094)$ & 0,676 \\
\hline \multicolumn{5}{|l|}{ Estado nutricional $^{*}$} \\
\hline Eutrofia & 71 & 35,3 & - & 1,0 \\
\hline Baixo peso & 42 & 38,2 & $0,000(2,356-5,251)$ & 3,517 \\
\hline Sobrepeso/obesidade & 146 & 65,8 & $0,000(1,936-4,997)$ & 3,110 \\
\hline \multicolumn{5}{|l|}{ No doenças } \\
\hline Nenhuma & 39 & 40,6 & - & 1,0 \\
\hline 1 & 62 & 45,6 & $0,030(1,068-3,672)$ & 1,980 \\
\hline 2 ou 3 & 121 & 51,1 & $0,101(0,911-2,870)$ & 1,617 \\
\hline 4 ou mais & 42 & 57,5 & $0,333(0,765-2,205)$ & 1,299 \\
\hline \multicolumn{5}{|l|}{ Percepção de saúde } \\
\hline Boa & 79 & 45,1 & - & 1,0 \\
\hline Ruim & 185 & 50,4 & $0,252(0,564-1,162)$ & 0,810 \\
\hline \multicolumn{5}{|l|}{ AFR } \\
\hline Sim & 50 & 50,5 & - & 1,0 \\
\hline Não & 214 & 48,3 & $0,692(0,706-1,688)$ & 1,092 \\
\hline
\end{tabular}

*Associação estatisticamente significativa entre PIC e estado nutricional ( $p<0,001)$. 
Tabela 3. Modelo de regressão logística univariada em relação ao desfecho percepção da imagem corporal insatisfeito e fatores associados [estado nutricional, no de doenças, percepção de saúde, prática de atividade física regular (AFR)] entre os homens. Campina Grande (PB), Brasil.

\begin{tabular}{|c|c|c|c|c|}
\hline Variáveis & $\mathbf{n}$ & $\begin{array}{r}\text { Percepção da imagem } \\
\text { corporal insatisfeita (\%) }\end{array}$ & p (IC 95\%) & OR \\
\hline Grupo etário* & 35 & 36,1 & - & 1,0 \\
\hline 60 a 69 anos & 33 & 37,9 & $0,009(0,146-0,757)$ & 0,332 \\
\hline $\begin{array}{l}70 \text { a } 79 \text { anos } \\
80 \text { anos ou mais }\end{array}$ & 9 & 15,8 & $0,005(0,133-0,706)$ & 0,307 \\
\hline Estado nutricional ${ }^{*}$ & 31 & 25,4 & - & 1,0 \\
\hline Eutrofia & 14 & 25,0 & $0,000(1,938-7,417)$ & 3,792 \\
\hline $\begin{array}{l}\text { Baixo peso } \\
\text { Sobrepeso/obesid }\end{array}$ & 31 & 56,4 & $0,001(1,731-8,677)$ & 3,875 \\
\hline No doenças & 17 & 23,6 & - & 1,0 \\
\hline Nenhuma & 23 & 29,5 & $0,084(0,882-7,599)$ & 2,588 \\
\hline 1 & 29 & 39,7 & $0,226(0,670-5,463)$ & 1,913 \\
\hline $\begin{array}{l}2 \text { ou } 3 \\
4 \text { ou mais }\end{array}$ & 8 & 44,4 & $0,715(0,429-3,438)$ & 1,214 \\
\hline Percepção de saúde & 34 & 30,9 & - & 1,0 \\
\hline $\begin{array}{l}\text { Boa } \\
\text { Ruim }\end{array}$ & 43 & 32,8 & $0,751(0,531-1,578)$ & 0,916 \\
\hline AFR & 23 & 42,6 & - & 1,0 \\
\hline $\begin{array}{l}\text { Sim } \\
\text { Não }\end{array}$ & 54 & 28,9 & $0,059(0,978-3,415)$ & 1,827 \\
\hline
\end{tabular}

"Associação estatisticamente significativa entre PIC, grupo etário e estado nutricional $(\mathrm{p}<0,001)$.

homens. Observa-se que a maior prevalência de insatisfação com a imagem corporal esteve entre aqueles com 60 a 69 anos $(36,1 \%)$, sobrepeso/obesidade $(56,4 \%)$, que referiram presença de quatro ou mais doenças $(44,4 \%)$, com percepção da saúde ruim $(32,8 \%)$ e com prática de atividade física regular (42,6\%). Foi verificada associação estatisticamente significativa entre percepção da imagem corporal e grupo etário e estado nutricional.

Entre os homens, tanto aqueles com baixo peso como os com sobrepeso/obesidade apresentavam risco aumentado de estarem insatisfeitos com sua imagem corporal quando comparados aos eutróficos, onde os com baixo peso apresentavam 3,79 vezes mais chances e os com sobrepeso/obesidade 3,87 vezes mais chances de insatisfação com a imagem.

Com relação aos resultados da regressão logística multivariada para idosos que se sentiam insatisfeitos em relação à percepção da sua imagem corporal, observou-se que, entre as mulheres, foram incluídas no modelo as variáveis, estado nutricional e número de doenças crônicas, as quais apresentaram associação significativa com a percepção da imagem corporal. Após serem testados os modelos permaneceu entre as mulheres apenas a variável estado nutricional. Do mesmo modo foi feito entre os homens, tendo sido incluídas no modelo multivariado as variáveis grupo etário, estado nutricional e número de doenças crônicas. Após serem testadas as variáveis para construção do modelo, permaneceram as variáveis grupo etário e estado nutricional. Na Tabela 4 é apresentado apenas o modelo de regressão multivariado para os homens, visto que entre as mulheres apenas uma variável permaneceu estatisticamente significativa (estado nutricional) (Tabela 2).

Dessa forma, é possível observar que ser homem em idades mais avançadas constituiu fator de proteção para insatisfação com a imagem corporal, tendo em vista que os idosos dos grupos etários 70 a 79 anos e 80 anos ou mais apresentaram chances 2,50 e 2,58 inferiores, respectivamente, de se sentirem insatisfeitos com sua imagem corporal que os idosos com 60 a 69 anos. $\mathrm{O}$ estado nutricional não adequado mostrou-se como fator de risco para insatisfação com a imagem corporal, tanto o baixo peso $(\mathrm{OR}=3,848)$ como o sobrepeso/obesidade $(\mathrm{OR}=3,773)$, quando comparados aos eutróficos. 


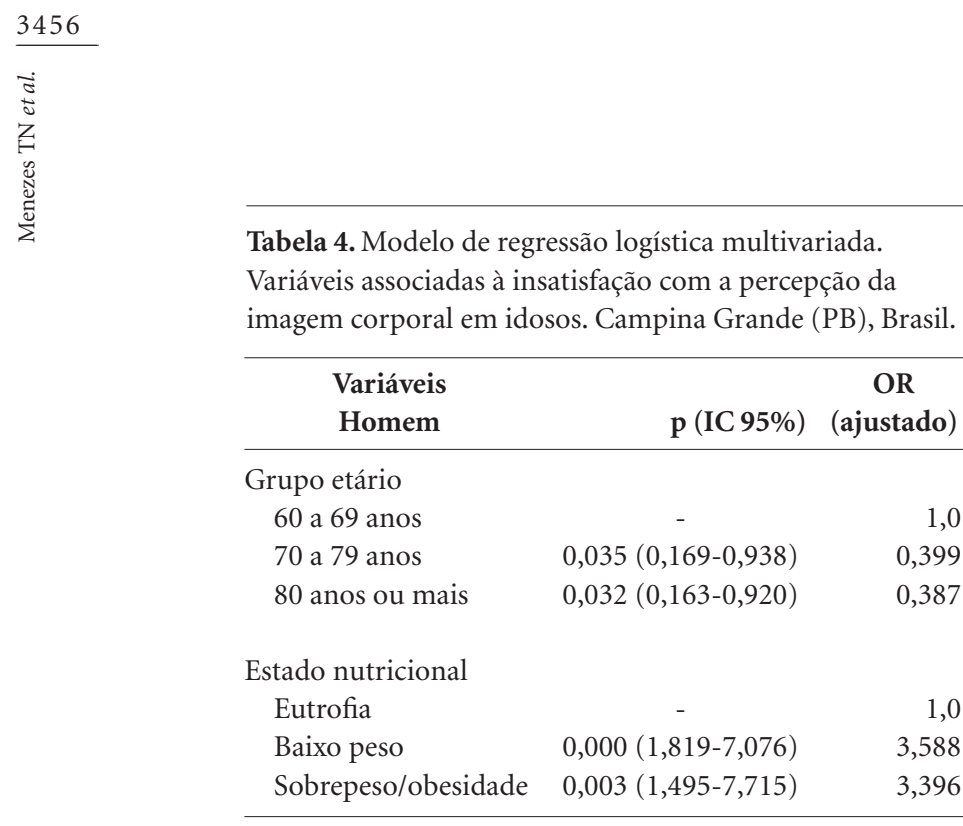

\section{Discussão}

A percepção da imagem corporal pode influenciar a saúde dos indivíduos, tendo em vista que muitos comportamentos relacionam-se diretamente ao modo de se perceber externamen$\mathrm{te}^{12,27,28}$. Verificar como o indivíduo percebe o seu corpo permite observar o estado de satisfação ou não com a sua aparência física ${ }^{14} \mathrm{e}$ a partir disso identificar possíveis comportamentos de risco relacionados à saúde. Essa percepção do indivíduo sobre sua imagem corporal pode ser verificada por meio da observação da escala de silhuetas, a qual consiste em um método rápido, simples e de boa fidedignidade ${ }^{21}$.

Neste estudo, observou-se que a maior proporção, tanto de homens como de mulheres, referiu satisfação com a sua imagem corporal, o que pode refletir uma melhor aceitação da sua imagem corporal frente ao envelhecimento, contribuindo significativamente para a autoestima, favorecendo assim o autocuidado e repercutindo na busca por um melhor estado de saúde.

Essa maior proporção de idosos satisfeitos com a imagem corporal foi também verificada em estudo realizado com participantes de programas de exercício físico de duas universidades públicas no Sul do Brasil, o qual verificou que $70,0 \%$ dos idosos apresentavam satisfação com a autoimagem. Essa satisfação esteve relacionada à percepção positiva da aparência física e da capacidade funcional, a condições satisfatórias de vida, à aceitação/adaptação à idade, a condições financeiras favoráveis, ao bom relacionamento com os filhos, ao contato social e ao desejo de estudar. Todavia, o instrumento utilizado no ci- tado estudo foi o questionário de autoimagem e autoestima de Steglich ${ }^{18}$.

Como visto, a percepção satisfatória da imagem corporal pode tanto ser influenciada como repercutir em diversos aspectos da vida do indivíduo, o que pode intervir positivamente no estado de saúde dos indivíduos.

Estudos verificando elevada proporção de idosos satisfeitos com a imagem corporal, não são comuns, tendo em vista que os resultados da maioria dos estudos consultados mostraram elevada prevalência de insatisfação entre ido$\operatorname{sos}^{4,7,10,12,16,20}$, principalmente para o sexo feminino ${ }^{14,20}$. Essa insatisfação com a imagem corporal entre idosos está relacionada à não aceitação do processo de declínio funcional do organismo e à impossibilidade de reversão do processo de envelhecimento, assim como à exigência social por um tipo físico ideal estabelecido ${ }^{29}$. Essa não aceitação das modificações ocorridas com o corpo devido ao envelhecimento é preocupante, pois podem desencadear mecanismos compensatórios não saudáveis que coloquem em risco a saúde dos indivíduos.

Foi verificado neste estudo que os homens apresentaram-se mais satisfeitos com sua imagem corporal que as mulheres, o que pode ser justificado pela menor influência da mídia sobre o padrão de beleza masculino ${ }^{30} \mathrm{e}$ maior influência sobre o padrão de beleza feminino $0^{5,31,32}$. Contudo, tanto homens como mulheres podem apresentar distorção na percepção da imagem corporal, seja subestimando-a ou superestimando- $\mathrm{a}^{33}$, porém poucos são os estudos que têm buscado identificar a satisfação com a imagem corporal dos indivíduos do sexo masculino $0^{30,34}$.

No presente estudo foi observado, ainda, que idosos mais velhos (80 anos ou mais) apresentaram maior satisfação com a imagem do seu corpo que idosos mais novos. Esse achado corrobora com pesquisa realizada por Anderson et al..$^{14}$ que verificou maior prevalência de satisfação com a imagem corporal em idosos mais velhos quando comparados aos idosos mais novos. Estudo realizado por Meurer et al..$^{18} \mathrm{em}$ estado do Sul do Brasil verificou que idosos com 80 anos ou mais apresentaram melhor autoimagem e que provavelmente essa satisfação, nesse grupo etário, estaria relacionada à aceitação das mudanças inerentes ao envelhecimento. A elevada satisfação com a imagem corporal em idosos mais velhos pode ser explicada pelo fato de que nessa faixa etária os indivíduos se preocupam mais com a saúde e a funcionalidade do organismo do que com a estética $^{35}$. 
Apesar de estudos com idosos mostrarem a associação da percepção da imagem corporal com o número de doenças ${ }^{11}$, percepção da saúde ${ }^{12}$ e atividade física regular ${ }^{1,9}$, neste estudo, tanto em homens como em mulheres, essa associação não foi observada. No entanto, há que se considerar a elevada prevalência de idosos insatisfeitos apresentando maior número de doenças, percepção da saúde ruim e com prática de atividade física regular. Fato importante tendo em vista que a satisfação ou não com a imagem corporal pode ser um forte indicador de mortalidade ${ }^{36}$, além de estar associada ao reconhecimento pelo idoso das suas particularidades fisiológicas, psicológicas e sociais ${ }^{1}$, como também do seu estado de saúde ${ }^{10}$.

Observando as associações entre percepção da imagem e o estado nutricional do idoso, este estudo verificou que a maior prevalência de insatisfação com o corpo entre as mulheres esteve relacionada ao sobrepeso/obesidade. Essa elevada prevalência também foi observada em pesquisa realizada por Pereira et al. ${ }^{17}$, na qual $72,6 \%$ das mulheres idosas apresentavam descontentamento com a sua imagem corporal em decorrência do excesso de peso. Resultado semelhante foi observado em pesquisa realizada em Santa Maria/RS onde a prevalência de insatisfação com a imagem corporal de mulheres pelo excesso de peso foi de $84,4 \%{ }^{37}$. Pode-se considerar que essa insatisfação com a imagem corporal será positiva caso a mesma gere um estímulo à adoção de hábitos de vida saudáveis que busquem um estado nutricional adequado. Porém, será negativa se essa insatisfação gerar baixa autoestima, ansiedade, depressão e a adoção de comportamentos de risco objetivando alcançar o peso ideal.

Neste estudo foi observado, ainda, que mulheres com baixo peso apresentaram considerável proporção de insatisfação com sua imagem corporal. Esse resultado confirma os achados da pesquisa realizada por Tribess et al. ${ }^{10}$ que verificou elevada prevalência de idosas com baixo peso insatisfeitas com a sua imagem corporal $(88,9 \%)$. Esses autores consideram que a forte tendência cultural por um corpo magro e aceitável socialmente pode ser um dos motivos para a insatisfação entre mulheres, mesmo entre aquelas com baixo peso. Essa insatisfação com o peso corporal pode estar relacionada à pressão por determinados padrões de beleza que tem dificultado a percepção ou aceitação pelas mulheres da sua própria imagem corporal, desencadeando uma compulsão por uma anatomia ideal, levando-as a evitar situações do cotidiano para não expor o corpo devido à insatisfação ${ }^{37}$.
É preciso identificar o motivo pelo qual as idosas se encontram insatisfeitas com a imagem corporal, pois a partir dessa informação é possível estabelecer estratégias que proporcionem o alcance do estado nutricional adequado gerando assim satisfação com sua imagem.

Com relação aos homens, verificou-se que a maior prevalência de insatisfação com o corpo esteve relacionada à idade e ao estado nutricional. Observou-se que idosos mais jovens apresentaram maior prevalência de insatisfação com sua imagem corporal quando comparados aos idosos mais velhos. Apesar de na literatura consultada não terem sido encontrados estudos específicos com idosos do sexo masculino, no estudo de Meurer et al. ${ }^{18}$ realizado com idosos de ambos os sexos, observou-se que idosos mais velhos apresentavam melhor autoimagem do que os mais novos, provavelmente pela própria aceitação adquirida com o tempo.

Foi verificado, ainda, que a maior prevalência de insatisfação com o corpo entre os homens esteve relacionada ao estado nutricional. A insatisfação em relação ao excesso de peso foi observada em menor proporção nos homens quando comparados às mulheres. De acordo com McCreary e Sasse $^{38}$ e Pope et al. ${ }^{34}$, diferentemente dos padrões de magreza estabelecidos para as mulheres, as pressões culturais sofridas pelos homens em relação à composição corporal incentivam a busca de um corpo forte, musculoso ou volumoso. No entanto, essa menor insatisfação com a imagem corporal dos homens quando comparados às mulheres, pode também estar associada a uma tendência de subestimação de suas dimensões corporais, não dependendo do estado nutricional apresentado ${ }^{33}$. Esse fato chama atenção, pois esse indivíduo estará em risco para o desenvolvimento de alguns agravos em decorrência do sobrepeso/obesidade, visto que estando satisfeito com essa condição, não buscará auxílio para alcançar um estado nutricional adequado.

Observa-se que, apesar de ter sido encontrada maior proporção de homens satisfeitos com a sua imagem corporal relacionado ao estado nutricional quando comparados às mulheres, $\mathrm{o}$ risco para apresentar insatisfação com a imagem corporal foi maior entre esses indivíduos que entre as mulheres, quando se considera o estado nutricional não adequado. Dessa forma, é possível afirmar que o estado nutricional inadequado está associado à insatisfação da imagem corporal independente do sexo.

Diante disso, faz-se necessário reconhecer a importância da percepção da imagem corporal e 
sua relação com o estado nutricional durante a implementação de estratégias para a promoção da saúde do idoso, tendo vista que idosos podem estar satisfeitos com sua imagem corporal mesmo apresentando estado nutricional inadequado, ou podem estar insatisfeitos com sua imagem, porém com estado nutricional adequado. Fazer diferenciação entre esses dois grupos possibilitará estratégias mais específicas, visto que os eutróficos devem ser estimulados a permanecerem com o peso ideal, além de se tornarem satisfeitos com seu estado. Por outro lado, os idosos com baixo peso ou sobrepeso/obesidade devem reconhecer as implicações desta inadequação no seu estado de saúde e devem ser estimulados na mudança de comportamentos a fim de alcançar o peso ideal, diminuindo, assim, os fatores de riscos relacionados ao estado nutricional inadequado.

\section{Considerações finais}

A imagem corporal envolve um complexo conjunto de fatores biopsicossociais que determinam subjetivamente como os indivíduos se vêem. Um dos fatores que influencia fortemente a percepção do corpo é o estado nutricional (aspecto biológico), como observado neste estudo, tendo em vista que o estado nutricional não adequado interferiu negativamente na percepção da imagem corporal dos idosos de ambos os sexos deste estudo. Sendo assim, deve-se estimular mudanças que possibilitem a adequação do estado nutricional com consequente aumento da satisfação com imagem corporal entre os idosos. Estar bem com a sua imagem corporal é algo positivo, principalmente se o indivíduo estiver com estado nutricional adequado.

Diante da compreensão de que o modo como o indivíduo se percebe pode determinar mudanças positivas ou negativas nos hábitos relacionados ao estilo de vida, cabe aos profissionais de saúde identificar possibilidades de atuação a partir da percepção que os idosos têm do seu corpo, reconhecendo a importância da percepção da imagem corporal e sua relação com o estado nutricional e, a partir dessas informações, estabelecer estratégias a fim de que os mesmos adotem hábitos saudáveis e mantenham a satisfação com sua imagem corporal.

Uma das limitações desse estudo relaciona-se ao instrumento utilizado para verificar a percepção da imagem corporal dos idosos, tendo em vista que as imagens apresentadas são bidimensionais, o que pode dificultar a compreensão da figura observada. Sugerimos a construção de um instrumento que utilize imagens tridimensionais que facilite a percepção antropométrica do corpo e uma maior fidedignidade na avaliação.

Uma dificuldade percebida na elaboração desta pesquisa foi a escassez de estudos com essa temática realizados com a população do sexo masculino, o que dificultou a comparação com os achados deste estudo. Dessa forma, sugere-se a realização de estudos que objetivem esclarecer de que forma o homem percebe a sua imagem corporal e os fatores que a influenciam. Além de estudos que identifiquem o motivo pelo qual os idosos se encontram insatisfeitos com a sua imagem corporal, considerando outros aspectos como psicológicos, sociais e culturais.

\section{Colaboradores}

TN Menezes participou do delineamento, coordenação e orientação do estudo, análise, interpretação e discussão dos dados e da revisão final do artigo. KQD Brito e ECT Oliveira participaram da interpretação e discussão dos dados e da redação do artigo. DF Pedraza contribuiu na interpretação e discussão dos dados e na revisão do artigo. 


\section{Referências}

1. Fugulin BF, Rosche S, Resende R, Rossi L. Prática de Atividade Física e Autoimagem de Idosas. CERES 2009; 4(2):57-64.

2. Nascimento LMP, Amaral RM, Menezes RL, Sandoval RA. Percepção da imagem corporal, auto-estima e qualidade de vida em alunos da UNATI/UCG. Lecturas, Educación Física y Deportes, Revista Digital 2008; 13(127).

3. Rusch SGS, Mallet LG, Wendt GW, Argimon IL. Opções de lazer e atividades físicas e sua relação com a qualidade de vida em idosos. Movimento \& Percepção 2008; 9(12):62-76

4. Chaim J, Izzo H, Sera CTN. Cuidar em saúde: satisfação com imagem corporal e autoestima de idosos. O mundo da saúde 2009; 33(2):175-181.

5. Damasceno VO, Vianna VRA, Vianna JM, Lacio M, Lima JRP, Novaes JS. Imagem corporal e corpo ideal. Rev Bras Cien e Mov 2006; 14(2):81-94.

6. Santos SALD, Tavares DMDS, Barbosa MH. Fatores socioeconômicos, incapacidade funcional e número de doenças entre idosos. Rev Eletrônica de Enfermagem 2010; 12(4):692-697.

7. Machado DC, Sudo N, Pinto AHG. Imagem corporal de idosas que residem em uma instituição de longa permanência de Porto Alegre-RS. CERES 2010; 5(3):139-148.

8. Rocha MP, Viebig RF, Latterza AR. Imagem corporal em idosos: influências dos hábitos alimentares e da prática de atividade física. Lecturas, Educación Física y Deportes, Revista Digital 2012; 15(166).

9. Fermino RC, Pezzini MR, Reis RS. Motivos para prática de atividade física e imagem corporal em frequentadores de academia. Rev Bras Med Esporte 2010; 16(1):18-23.

10. Tribess S, Virtuoso Jr JS, Petroski EL. Estado nutricional e percepção da imagem corporal de mulheres idosas residentes no nordeste do Brasil. Cien Saude Colet 2010; 15(1):31-38.

11. Teixeira JS, Corrêa JC, Rafael CBS, Miranda VPN, Ferreira MEC. Envelhecimento e Percepção Corporal de Idosos Institucionalizados. Rev Bras Geriatr Gerontol 2012; 15(1):63-68.

12. Leal SA. Estado de saúde auto-percebido, índice de massa corporal e percepção da imagem corporal em utentes dos cuidados de saúde primários [dissertação]. Lisboa: Universidade de Lisboa; 2009.

13. Reboussin BA, Rejeski WJ, Martin KA, Callahan K, Dunn AL, King AC, Sallis JF. Correlates of satisfaction with body function and body appearance in middle - and older aged adults: the activity counseling trial (ACT). Psych and Health 2000; 15(2):239-254.

14. Anderson LA, Eyler AA, Galuska DA, Brown DR, Brownson RC. Relationship of satisfaction with body size and trying to lose weight in a national survey of overweight and obese women aged 40 and older, United States. Prev Med 2002; 35(4):390-396.

15. Baker L, Gringart E. Body image and self-esteem in older adulthood. Ageing Soc 2009; 29(6):977-995.

16. Barbosa RMSP, Cabral FN, Brandão SAF, Neri JL. Envelhecimento: relações da imagem corporal com os parâmetros peso e altura. BIUS 2011; 2(2):4-16.

17. Pereira EF, Teixeira CS, Borgatto AF, Daronco LSE. Relação entre diferentes indicadores antropométricos e a percepção da imagem corporal em idosas ativas. Rev Psiq Clín 2009; 36(2):54-59.
18. Meurer ST, Beneditti TRB, Mazo GZ. Aspectos da autoimagem e autoestima de idosos ativos. Motriz 2009; 15(4):788-796.

19. Damasceno VO, Lima JRP, Vianna JM, Vianna VRA, Novaes JS. Tipo físico ideal e satisfação com a imagem corporal de praticantes de caminhada. Rev Bras Med Esporte 2005; 11(3):181-186.

20. Moura PV, Freitas CMSM, Silva EAPC, Silva PPC. Ser idoso: a imagem corporal no envelhecer. I Congresso Brasileiro de Imagem Corporal; 2010 Ago 9-10; Campinas (SP): Unicamp; 2010

21. Coqueiro RS, Petroski EL, Pelegrini A, Barbosa AR. Insatisfação com a imagem corporal: avaliação comparativa da associação com estado nutricional em universitários. Rev Psiquiatr Rio Gd. Sul 2008; 30(1):31-168.

22. Matsuo RF, Velardi M, Brandão MRF, Miranda MLJ. Imagem corporal de idosas e atividade física. REFEME 2007; 6(1):37-43.

23. Araújo DSMS, Araújo CGS. Self-perception and dissatisfaction with weight does not depend on the frequency of physical activity. Arq Bras Cardiol 2003; 80(3):235-249.

24. Stunkard AJ, Sorenson T, Schlusinger F. Use of the Danish Adoption Register for the study of obesity and thinness. In: Kety SS, Rowland LP, Sidman RL, Matthysse SW, editors. The genetics of neurological and psychiatric disorders. New York: Raven; 1983. p. 115-120.

25. Lebrão ML, Duarte YAO. Sabe - saúde, bem-estar e envelhecimento - O Projeto Sabe no município de São Paulo: uma abordagem inicial. Brasília: Organização Pan-Americana da Saúde; 2003.

26. Gordon CC, Chumlea WC, Roche AF. Stature, recumbent length, and weight. In: Lohman TG, Roche AF, Martorell $\mathrm{R}$, editors. Anthropometric standardization reference manual. Champaign: Human Kinetics Books; 1988. p. 3-8.

27. Silva JD, Silva ABJ, Oliveira AVK, Nemer ASA. Influência do estado nutricional no risco para transtornos alimentares em estudantes de nutrição. Cien Saude Colet 2012; 17(12):3399-3406

28. Petroski EL, Pelegrini A, Glaner MF. Motivos e prevalência de insatisfação com a imagem corporal em adolescentes. Cien Saude Colet 2012; 17(4):1071-1077.

29. Fonseca CC, Gama EF, Thurm BE, Pereira ES, Limongelli AMA, Miranda MLJ. Benefícios da estimulação perceptual corporal no esquema corporal de idosos. Rev Bras Geriatr Gerontol 2012; 15(2):353-364.

30. Vieira FR, Lacerda Y, Dantas EEM, Novaes IS. Efeitos das Atividades Físicas em Academias na Imagem Corporal dos Obesos. Fitness \& Perform J 2005; 4 (1):19-26.

31. Bedford JL, Johnson CS. Societal influences on body image dissatisfaction in younger women.J Women Aging 2006; 10(1):41-55

32. Maldonado GR. A educação física e o adolescente: a imagem corporal e a estética da transformação na mídia impressa. Rev Mack de Educ Fís e Esporte 2006; 5(1):59-76.

33. Kakeshita IS, Almeida SS. Relação entre índice de massa corporal e a percepção da auto-imagem em universitários. Rev Saude Publica 2006; 40(3):497-504.

34. Pope HG, Gruber AJ, Mangweth B, Bureau B, deCol C, Jouvent R, Hudson JI. Body Image Perception Among Men in Three Countries. Am J Psychiatry 2000; 157(8):1297-1301. 
35. Alves LC, Rodrigues RN. Determinantes da autopercepção de saúde entre idosos do Município de São Paulo, Brasil. Rev Panam Salud Publica 2005; 17(5/6):333-341.

36. Bevilacqua LA, Daronco LSE, Balsan LAG. Fatores associados à insatisfação com a imagem corporal e autoestima em mulheres ativas. Salusvita 2012; 31(1):55-69.

37. Mclaren L, Kuh D. Body dissatisfaction in midlife women. J Women Aging 2004; 16(1-2):35-54.

38. McCreary DR, Sasse DK. An exploration of the drive for muscularity in adolescent boys and girls. J Am Coll Health 2000; 48(6):297-304.

Artigo apresentado em 24/08/2013

Aprovado em 16/11/2013

Versão final apresentada em 23/11/2013 